\title{
On the nature of the chromospheric fine structure
}

\section{Intensity and velocity oscillations of dark mottles and grains}

\author{
K. Tziotziou ${ }^{1, \star}$, G. Tsiropoula ${ }^{1}$, and P. Mein ${ }^{2}$ \\ 1 National Observatory of Athens, Institute for Space Applications and Remote Sensing, Lofos Koufos, \\ 15236 Palea Penteli, Greece \\ e-mail: [kostas; georgia]@space.noa.gr \\ 2 Observatoire de Paris, Section de Meudon, LESIA, 92195 Meudon Principal Cedex, France \\ e-mail: pierre.mein@obspm.fr
}

Received 11 February 2003 / Accepted 10 May 2004

\begin{abstract}
We study periodicities of dark mottles and grains observed in high spatial and temporal resolution $\mathrm{H} \alpha$ 2-D intensity and velocity images, obtained with the Multichannel Subtractive Double Pass (MSDP) spectrograph at THEMIS in Tenerife, Canary Islands. Intensity and velocity variations of individual mottles and grains, as well as of large regions containing a substantial number of these structures are examined with a wavelet analysis which provides the temporal distribution of periods and with a randomization method that gives the respective probabilities associated with them. The wavelet analysis, which shows that velocity variations are the dominant ones, results in a wide range of periods, from 100 to $500 \mathrm{~s}$, with variable probabilities. However, it strongly indicates the presence of a predominant period of $\sim 5 \mathrm{~min}$ seen in both dark mottle and grain oscillations. We suggest that differences in periods found in individual mottles as well as in averages of regions containing these structures are due to the topology of the local magnetic field which can affect oscillatory processes. We report for the first time oscillations of dark grains which are more likely vertical structures "anchored" in the photosphere. The analysis supports further the suggestion that dark mottles and grains are the same structures, the only difference between them being their respective inclinations to the line-of-sight.
\end{abstract}

Key words. Sun: chromosphere - Sun: oscillations - methods: data analysis

\section{Introduction}

The solar chromosphere, especially when viewed in $\mathrm{H} \alpha$, shows a rich diversity of highly dynamical structures of different intensities, shapes and sizes. Due to this diversity there is a widespread tendency to distinguish phenomena occurring in active regions from those occurring in quiet ones. However, the key agent of all observed phenomena is the magnetic field distributed in different scales over the solar disk. Both quiet and active regions are considered to be made up by the same elementary magnetic flux tubes, their difference being primarily due to different filling factors (flux tubes are less packed in quiet regions) which result to different magnetic flux densities.

The dominant feature of the "quiet" solar chromosphere, when observed in strong lines, such as $\mathrm{Ca}$ II $\mathrm{H}$ and $\mathrm{K}$ and $\mathrm{H} \alpha$, is a large-scale cellular pattern known as the "chromospheric network". In the Ca II $\mathrm{K}$ line, bright elements outline the cell boundaries (CB) while in $\mathrm{H} \alpha$, especially in the wings of this line, several elongated dark or bright structures, called mottles, are observed. It is now well established that the chromospheric

^ Present address: Sterrekundig Instituut Utrecht, Postbus 80000, 3508 TA Utrecht, The Netherlands. network lies over the photospheric supergranular boundaries, where strong magnetic field concentrations are predominantly found swept there from the cell interiors (CI) by the convective motions.

In active regions the increased number density of magnetic flux tubes gives rise to the extended chromospheric plages which consist of many bright elements (referred to as facular granules) packed together. Extending out of the plages one can see bright and dark structures similar to the bright and dark mottles of the network, although, usually, more extended. These are referred to as fibrils. According to Foukal (1971) mottles and fibrils are similar structures, fibrils being more inclined than mottles relative to the line-of-sight (LOS) due to the different inclination of the local magnetic field. Morphological relationship between bright network and mottles is similar to the relation between plage and fibrils.

Due to the differences of magnetic fields the conditions in the $\mathrm{CI}$, at the $\mathrm{CB}$ and at the plages should differ considerably. Obviously, these differences must be reflected in the behaviour of the oscillations observed in these regions. Several authors have investigated intensity and velocity oscillations at the $\mathrm{CB}$ and in the $\mathrm{CI}$, while less work exists for plages. 


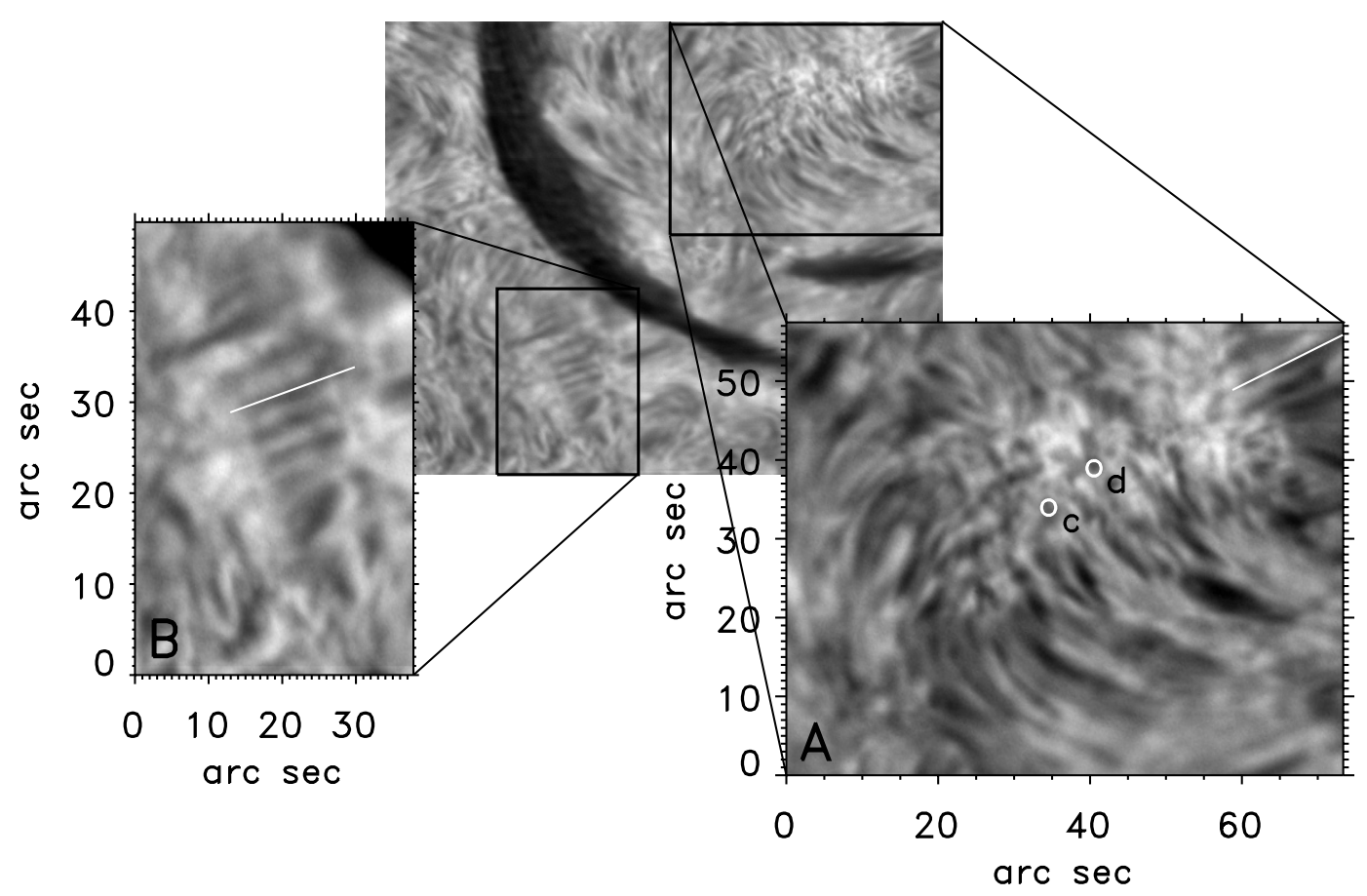

Fig. 1. An intensity image at $\mathrm{H} \alpha+0.24 \AA$ of the observed $150^{\prime \prime} \times 120^{\prime \prime}$ region. Enlargements show in more detail the two regions of interest A and B, where dark mottles and grains are mostly concentrated (see text and Paper I). The overplotted white lines correspond to the cuts along the mottles used for the time slice images of Fig. 2 (the feet of the mottles in Fig. 2 are the left and right end respectively of the cuts shown on region A and B). Letters "c" and "d" mark the position of the two individual dark grains used for this study.

The general findings of these works are that an enhanced power around $3 \mathrm{~min}$ is associated with the $\mathrm{CI}$, whereas at the $\mathrm{CB}$ the power spectra are dominated by oscillations with periods of the order or in excess of 5 min (Damé et al. 1984; von Uexküll et al. 1995; Deubner \& Fleck 1990; Lites et al. 1993). For a review see Rutten (1999). Recently, Hansteen et al. (2000) and Banerjee et al. (2001) reported that network oscillations are due to waves, which are produced in bursts with coherence time of about 10-20 min. As far as plages are concerned Bhatnagar \& Tanaka (1972) examining H $\alpha$ filtergrams have reported 5 min oscillations. Muglach (2003) using UV observations from the Transition Region and Coronal Explorer (TRACE) found $5 \mathrm{~min}$ oscillations in the plage and network regions and 3 min oscillations in the CI.

In Tziotziou et al. 2003 (hereafter Paper I) we reported observations of mottles and grains found in regions of low and high magnetic fluxes as observed with MDI on SOHO. Based on indirect evidence, we suggested that dark grains found close to a high magnetic flux region could be mottles the only difference between them being their inclination to the LOS, e.g. the first ones being almost vertical to the solar surface, while the latter having large inclinations relative to the LOS. In Paper I we have suggested a simple reconnection model explaining the pattern of bi-directional flows followed by downflowing material to explain the behaviour of observed velocities along mottles. We have also discussed the similarities between the quasiperiodic behaviour of the corresponding velocities in dark grains and mottles (Doppler and cloud velocities respectively).

The motivation for the present paper is to further study the dynamics of dark mottles and grains by exploring the periodic behaviour of intensity and velocity variations in different regions of the solar chromosphere where such structures reside and examining the differences or similarities. We take advantage of the two-dimensional information contained in $\mathrm{H} \alpha$ spectro-images and of the capabilities of the wavelet analysis, which allows the study of the duration of any statistically significant oscillations, as well as the derivation of their periods.

\section{Observations}

A $150^{\prime \prime} \times 120^{\prime \prime}$ field of view was observed in the $\mathrm{H} \alpha$ line on May 14th, 2000 with the MSDP spectrograph mounted on the French-Italian solar telescope THEMIS in Tenerife, Canary Islands. In Paper I we presented a description of the data acquisition and reduction procedure and a detailed dynamical and morphological study of dark mottles and grains. Below, we remind the reader of some of the basic points and characteristics of these observations.

Forty two-dimensional intensity images at $\mathrm{H} \alpha$ line-center, $\mathrm{H} \alpha \pm 0.24 \AA$ and $\mathrm{H} \alpha \pm 0.48 \AA$ as well as Doppler velocity images at $\mathrm{H} \alpha \pm 0.24 \AA$ and $\mathrm{H} \alpha \pm 0.48 \AA$, with a cadence of $\sim 40.5 \mathrm{~s}$, were obtained with the MSDP data reduction package. Figure 1 presents a sample $\mathrm{H} \alpha$ image showing a large filament bordered on each side by rows of elongated structures. On the top right part of the image (enlargement A of Fig. 1) the structuring of the area closer to the filament appears to be governed by it, while the structures away from the filament retain their radial orientation. The central region of this area contains several round-shaped dark structures having typical diameters 
of $1.5^{\prime \prime}-2^{\prime \prime}$. We called these structures dark grains in Paper I. A chain of mottles is formed below the main body of the filament (enlargement B of Fig. 1). In Paper I, based on the morphological similarity of region A to what is referred as a rosette in quiet regions, we refer to this region as a rosette. However, rosette interiors coincide with network cells, where magnetic fluxes are, generally, very low. Inspection of the $\mathrm{H} \alpha$ intensity images and the MDI magnetograms (see Fig. 3c of this paper and Fig. 4 of Paper I) shows that part of the bright central region coincides with a high - although rather diffuse - magnetic flux of the order of $100 \mathrm{G}$ characterizing plages (Schrijver 1987), while another part coincides with magnetic fluxes lower than $\sim 25 \mathrm{G}$. We shall refer from now on to the latter region, as the low magnetic flux plage region. Dark grains are found only in this region. We will also keep the term mottles for the dark elongated structures found around this central region, since their morphological characteristics, the downflows observed at their footpoints, as well as the temporal behaviour of the intensity and velocity are similar to those of mottles of region $\mathrm{B}$. We must note, however, that we have examined the velocity variations in some radial structures of region A and not in the curved ones.

\section{Data analysis}

A detailed discussion of the global properties of mottles based on the results of the cloud model approach was presented in Paper I. In this paper we are concentrating on the temporal behaviour of dark mottles, dark grains and the low magnetic flux plage region where these grains are located. We are examining the following parameters describing these structures: for dark mottles, a) the observed $\mathrm{H} \alpha$ line-center intensity and b) the velocity $v$ obtained from the cloud model; for dark grains and the low magnetic flux plage region, a) the observed $\mathrm{H} \alpha$ linecenter intensity and b) the Doppler velocity at $\mathrm{H} \alpha \pm 0.48 \AA$, because, as we explained in Paper I, the cloud model is not applicable in their case. It should be noted that use of these different methods for the derivation of the velocities will cause no discrepancies when comparing their variations, since as it has been shown (Tsiropoula 2000) i) cloud and Doppler LOS velocities have same signs, but different magnitudes the former being 2 to 4 times larger than the latter and ii) an almost linear relation exists between the cloud velocities and the Doppler velocities obtained at different wavelengths within the $\mathrm{H} \alpha$ profiles. We are also examining the temporal behaviour of individual dark mottles and grains and of entire regions containing dark mottles constituting the chromospheric network or found at the borders of the plage, as well as the low magnetic flux plage region where dark grains are located.

Analysis of individual structures. Temporal variations of physical parameters describing individual elongated structures like mottles, are better studied with time slice images, which show their distribution along the central axis of the structure as a function of time. In Fig. 2 we show the time slice images of $\mathrm{H} \alpha$ line-center intensity and cloud velocity along the central axes of the two mottles indicated in Fig. 1. We have averaged the respective physical parameters over a strip extending $0.3^{\prime \prime}$ on either side of the main axis of the mottles, which is carefully traced, taking into account a good co-alignment of the images of the time series. We have, furthermore, followed these axes from image to image of our time series and take into account small changes that occur in their direction, since, as we noticed in Paper I, individual mottles are slightly changing in direction in some frames. On the velocity time slice images we overplot some contours of downward (positive) and upward (negative) velocities, as well as the contour of zero velocity. For dark grains, which are small round shaped structures, the $\mathrm{H} \alpha$ line-center intensity and the Doppler velocity at $\mathrm{H} \alpha \pm 0.48 \AA$ are calculated as the spatial average of a $5 \times 5$ pixels square around the center of the grain, which is carefully traced in all images of the time series. It is clear from the time slice images and from Fig. 7 of Paper I that intensities and velocities along dark mottles and in dark grains are highly variable and that they have a repetitive character. As we have suggested in Paper I, these variations imply some kind of periodicity with periods of the order of $\sim 5 \mathrm{~min}$, which we will try to explore in this paper.

Selection process for a set of dark mottles. Distinguishing and tracing individual dark mottles in all images of the time sequence is not easy since their morphology changes with time. As a consequence a temporal analysis for all individual mottles of region $\mathrm{A}$ and $\mathrm{B}$, apart from time-consuming, is not always possible. However, we can perform an analysis of temporal variations of spatially averaged values of quantities, such as the calculated cloud velocities and observed intensities, describing mottles within an observed area. Pixels of these regions that belong to mottles are mainly selected for the spatial averaging by their intensity compared to the local background (see Paper I, Sect. 3 for selection criteria).

Selection process for different areas of region A. For the separation of different areas of region $A$ we follow a scheme similar to that adopted by Krijger et al. (2001). We have taken the average $\mathrm{H} \alpha$ line-center intensity per pixel over periods of $13.5 \mathrm{~min}$, which is half of the duration of our original time series, and we construct two 13.5 min intensity distributions. In Fig. $3 \mathrm{a}$ we show the resulting two distributions for region A of Fig. 1 in the form of histograms. Both distributions are almost the same and show a skewed non-Gaussian distribution with a high intensity tail. A pixel is assigned to be a mottles region if its average intensity is below the peak location in both distributions and as belonging to the plage region where no grains are present (hereafter grain-free region), if its intensity belongs to the high intensity tail in both histograms. Pixels that change category between distributions are excluded. Dark grains, are defined as pixels which are located inside the plage and have intermediate intensities between the two extremes taken for the mottles region and the grain-free region (hereafter grain region). Dark grain intensities form the small "bump" around 1250 counts in the intensity tail of both distributions. Figure $3 \mathrm{~b}$ shows the first $13.5 \mathrm{~min}$ average $\mathrm{H} \alpha$ linecenter intensity image of region A (shown in Fig. 1) with 

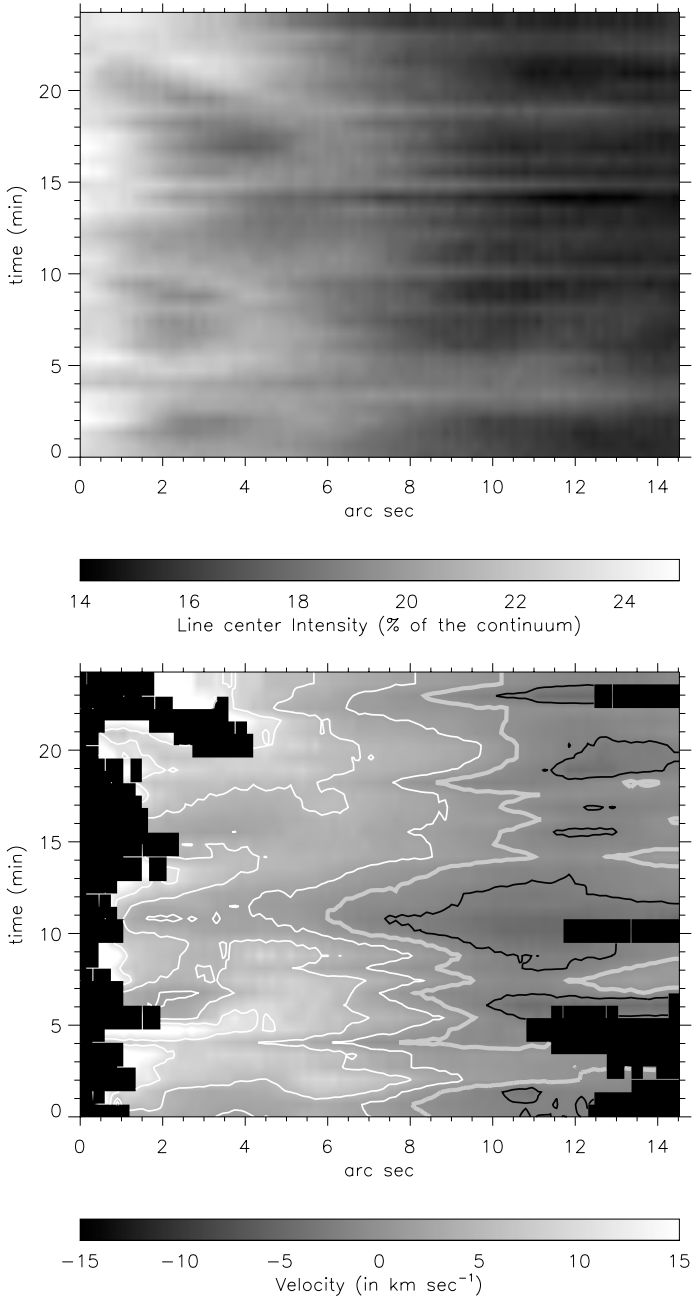
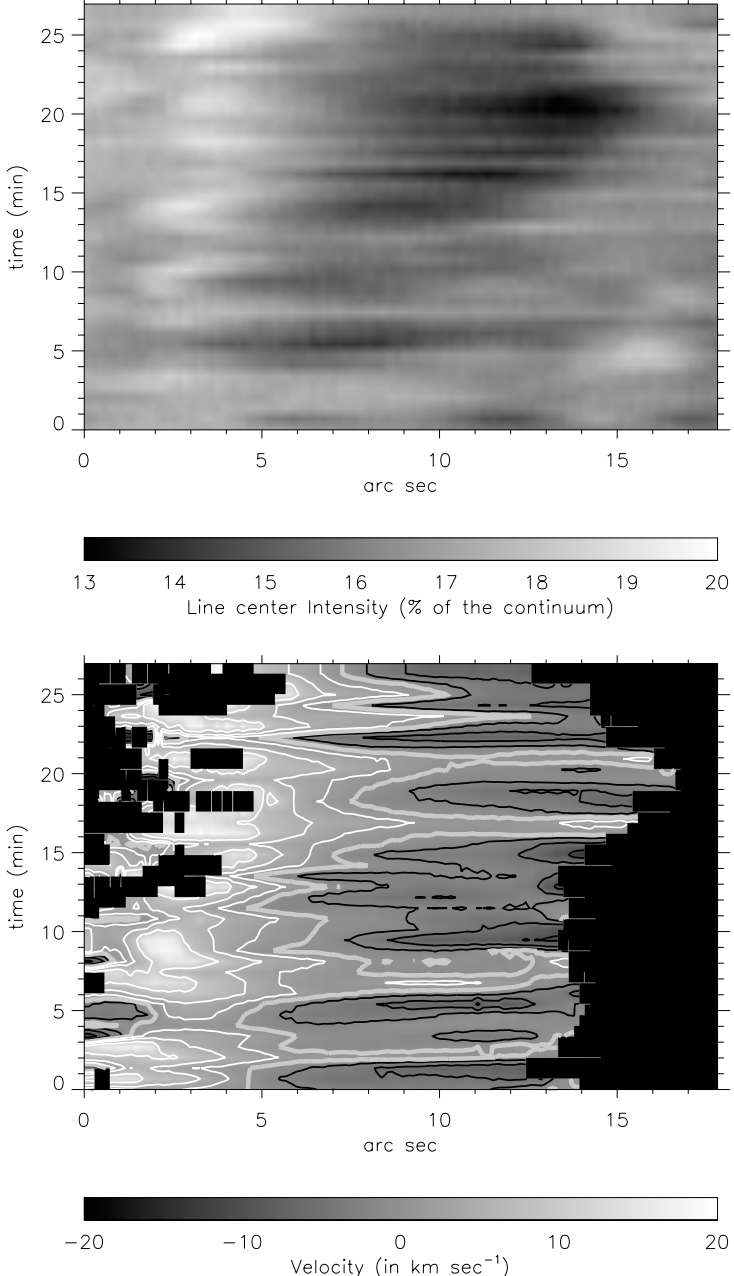

Fig. 2. Time slice images of $\mathrm{H} \alpha$ line-center intensity relative to the continuum (top row) and cloud velocity $v$ (bottom row) along a cut through a mottle of region A (left column) and region B (right column) indicated with white lines on the corresponding enlargements in Fig. 1. On the velocity time slice images, the black contours denote upward (negative) velocities, the white contours downward (positive) velocities, while the thick gray line represents the zero velocity contour. The left side of all images corresponds to the feet of the mottles (see Fig. 1).
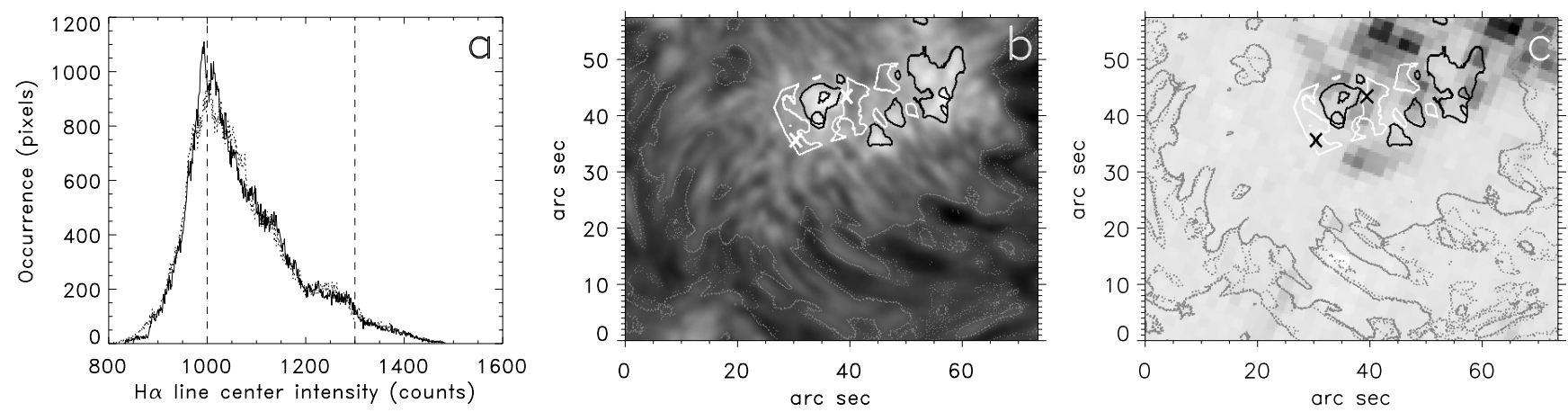

Fig. 3. Masks for the selection of different areas for region A of Fig. 1. a) Histograms of the number of pixels per line-center intensity bin in the two 13.5 min averages of region A (solid and dotted lines). Dashed lines show the division into mottles region (left strip), intermediate (middle strip) and grain-free region (right strip). Dark grains have intensities in the intermediate strip around the 1250 counts "bump". The first 13.5 min average b) $\mathrm{H} \alpha$ line-center intensity image and c) MDI fluxes (black corresponds to high magnitude fluxes) of region A with overlaid contours showing the mottles region (gray dotted contours), grain-free (thick solid black contours) and grain region (thick solid white contours) that resulted by taking into account both 13.5 min averages and also location in the case of dark grains (see text). The two " $x$ " in the grain region denote the position of the two individual grains "c" and "d" of Fig. 1. The grain and grain-free regions are found in the low magnetic flux plage region. 
overlaid contours showing the three regions that resulted from the aforementioned selection process. The same procedure can be followed for defining the mottles region in $\mathrm{B}$, which can be defined as pixels with average intensity below the peak location of both corresponding distributions.

In Fig. 3c we show the average per pixel MDI fluxes over a period of 13.5 min for region A (black corresponds to high magnitude fluxes) with overlaid contours showing the mottles region (gray dotted contours), the grain-free (black solid contours) and the grain region (black dotted contours). The magnitude of the spatial average magnetic flux for the three regions is respectively 5, 28 and $23 \mathrm{G}$. The grain-free and grain regions clearly have a considerable magnetic flux which is higher than the calculated noise $(\sim 8 \mathrm{G})$, but much less than the magnetic flux of the surrounding plage which is of the order of $100 \mathrm{G}$. There is no clear distinction between the magnetic fluxes of the grain-free and grain region. Individual structures can not be clearly associated with the accompanying magnetic field due to the low $\sim 2$ " spatial resolution of the MDI images.

\section{Description of the spectral analysis method}

Wavelet analysis. Periodic variations in physical quantities are usually analyzed by the Fourier or, more recently, by the wavelet transform of a time series. The wavelet analysis has the advantage that it decomposes a time series into the timefrequency space simultaneously, permitting the extraction of information on both the existence of any periodic signal within the series and how the period of the signal varies with time. For a detailed description of the wavelet analysis we refer the reader to the paper by Torrence \& Compo (1998), while a comparison between wavelet and Fourier analysis results of the same signal is performed by Fludra (2001). For the wavelet transform we use the Morlet wavelet, which consists of a plane wave modulated by a Gaussian. Since both time series and the wavelet function used are finite, edge effects due to zero padding are going to influence the wavelet transform. The cone of influence (hereafter COI), defines the region where these effects become important. Hence areas of the power spectrum in the COI are doubtful. In order to determine the significance level of any detected period, the data power spectrum is compared to the background noise spectrum. We assume that the noise in our time series $x_{\mathrm{n}}$ of $N$ data points is Poissonian $\left(\sigma_{\text {noise }}(x)=\sqrt{\bar{x}}\right.$, where $\left.\bar{x}=\frac{1}{N} \sum_{i=1}^{N} x_{i}\right)$ and choose a significance level of $95 \%$ which indicates that there is a $5 \%$ probability that any significant power is caused by chance.

The wavelet analysis returns the spectral power as a function of time and period. We define the global power spectrum as the average of the wavelet power spectrum over time. This averaging comprises the whole range of our time series, i.e. inside and outside the COI regions. The global power spectrum, which is only a function of period, is more or less equivalent to the Fourier analysis spectrum.

Statistical significance. The calculation of the statistical significance by comparison to an assumed distribution of random errors (e.g. Poisson) is obviously constrained by the choice of a specific noise model. Randomization tests, where the significance is calculated with respect to all possible arrangements of the observed data, are a more reliable approach, because they are distribution-free or non-parametric since no distributional assumptions of random errors are required. In this paper we use Fisher's method of randomization (Bradley 1968) as described in Nemec \& Nemec (1985) and applied by Banerjee et al. (2001). The method gives an estimate of the probability $p$ that no periodic component is present in the data by comparing the maximum power peak of $m$ (equal to 200 for our study) likely permutations of the time series data with the peak power of the original series. The probability levels shown in the plots of this paper are the percentage probability levels that a periodic component is present, that is the values of $(1-p) \times 100$. When the estimated $p$ value is zero, which means that there is an almost zero chance that the deduced period of the original series occurred by chance, the probability that the measured periodicity is real is equal to $96.5-100 \%$, since there is a maximum $0.5 m^{-1 / 2} \times 100$ standard error (Nemec \& Nemec 1985), equal to $3.5 \%$ in our case, for the estimated probability.

The randomization test described above can be applied as well to the global power spectrum, which represents the average spectral power for each period with time. In this case, a wavelet analysis is performed for $m$ equally likely permutations of the original time series data, the corresponding $m$ global power spectra are calculated and then the global probability is estimated by comparing the maximum power peak of these $m$ global power spectra, with the maximum power peak of the global power spectrum resulting from the original time series.

Due to the short duration of our series we restrict our analysis only to periods lower than $625 \mathrm{~s}$. We should also point out that only power peaks with a period lower than a maximum value (hereafter cut-off period) are considered for this randomization analysis. This period is defined as the maximum period of the COI line obtained from the wavelet analysis, which represents the maximum period of useful information that is not subjected to edge effects.

\section{Results}

\subsection{Intensity and velocity correlations in dark mottles and grains}

In Figs. 4a,b we have plotted the line-center intensity against the cloud velocity of the two dark mottles shown in region $\mathrm{A}$ and $\mathrm{B}$ and in Fig. 4c the line-center intensity against the Doppler velocity of the two dark grains "c" and "d" found in the grain region. Positive velocity values correspond to redshifted $\mathrm{H} \alpha$ profiles. For dark grains we considered all pixels that lie within the $5 \times 5$ pixels square around the center of the grain, which roughly corresponds to the surface area of the grain. We find that for the mottles there is a correlation between velocities and intensities, redshifts being usually correlated to high - but still below the chosen background (see Paper I) - intensities. This correlation is more clear for the mottle of region $\mathrm{A}$, found at the borders of the plage than for the mottle of region $\mathrm{B}$, which is found in a low magnetic flux 

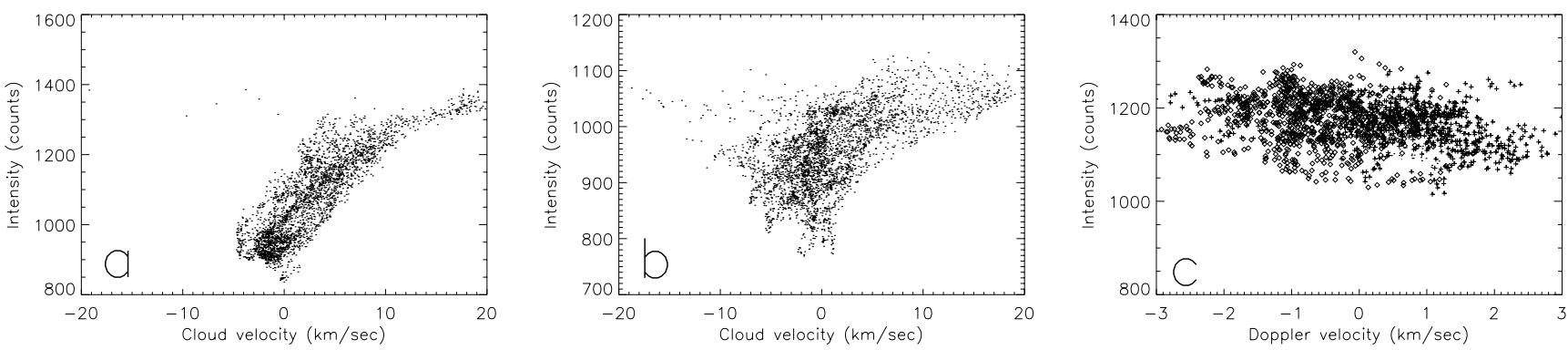

Fig. 4. The $\mathrm{H} \alpha$ line-center intensity versus a) cloud velocity for the mottle of region $\mathrm{A}, \mathbf{b}$ ) cloud velocity for the mottle of region $\mathrm{B}$ and c) Doppler velocity at $\mathrm{H} \alpha \pm 0.48 \AA$ for the two dark grains of Fig. 1 (crosses correspond to dark grain "c", while diamonds correspond to dark grain "d"). Downflow velocities are positive.
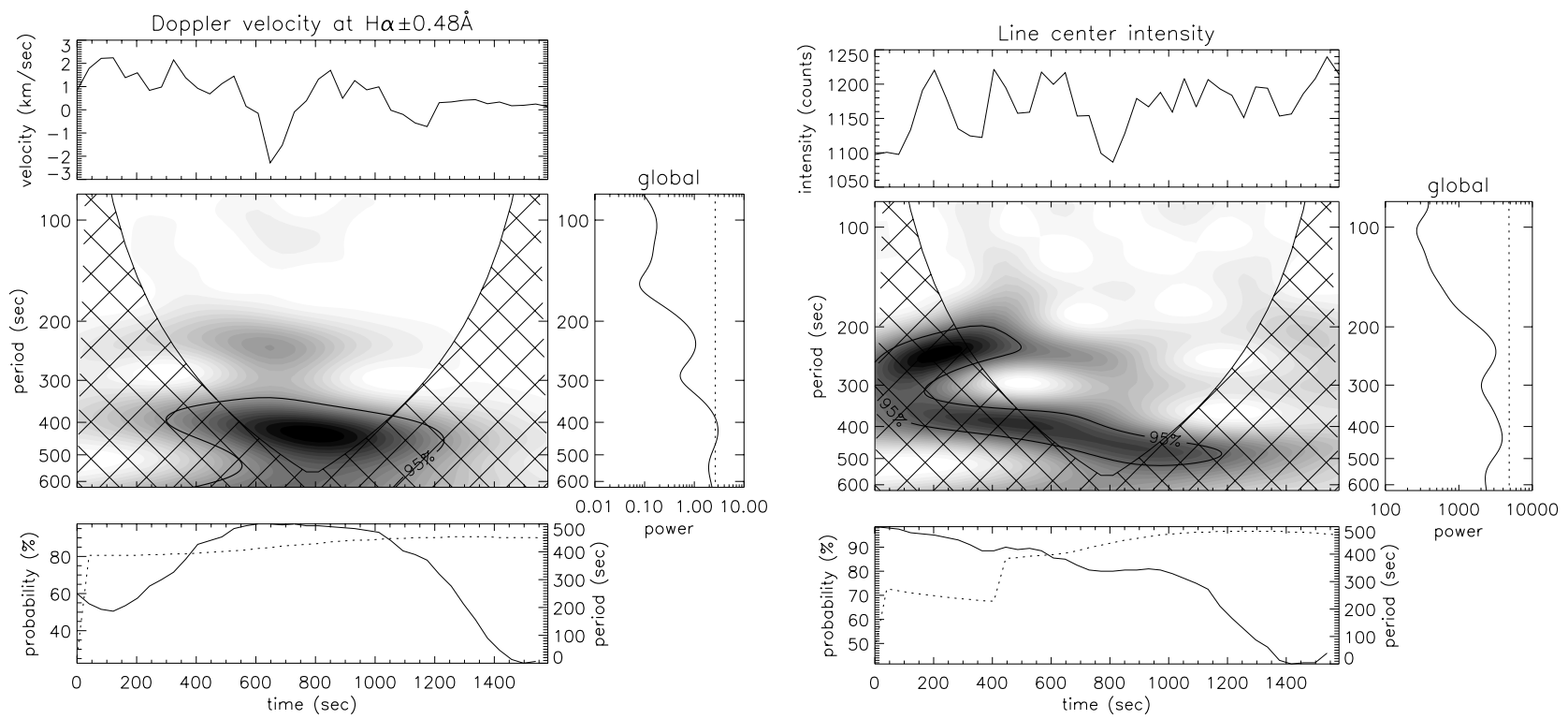

Fig. 5. Wavelet analysis results for the Doppler velocity (left column) and the H $\alpha$ line-center intensity variations (right column) for the dark grain "c" of Fig. 1. The middle left panels show the time-period calculated power spectra for the variations shown in the top panels with black representing high values of power (contours represent the significance level of 95\%). Cross-hatched regions indicate the COI regions. The middle right panels show the global power spectrum, i.e. the average of the wavelet power spectrum over time (the dotted line indicates the equivalent global significance level of 95\%). The lowest panels show the variations of the probability (solid line) obtained with the randomization method and the period (dotted line) of the maximum power peak below the cut-off period (562 s) corresponding to this probability. We should remind the reader that there is a standard 3.5\% error for the calculated probabilities. The respective peaks, below the cut-off period, of the global power spectra are at $424 \mathrm{~s}$ and $432 \mathrm{~s}$ with a corresponding global probability of $99 \%$ and $85 \%$.

region. This is consistent with the observational finding reported in Paper I (Sects. 2.2.1, 2.2.2), i.e. that downflows are observed at the roots of mottles, as well as brightness enhancements. On the other hand, there is no correlation between velocities and intensities of dark grains found in the grain region. It is interesting to note that searching for a correlation between intensity and velocity of transition region lines in CI and CB regions, Gallagher et al. (1999) and Hansteen et al. (2000) found that in the CB redshifts are correlated with high intensities, while no correlation exists between the intensity and the Doppler velocity in the CI.

In Paper I we have suggested that dark grains are essentially the same as dark mottles, the only difference between them being their respective inclinations relative to the LOS, with dark grains being almost vertical structures to the solar surface. If this is the case, then the lack of correlation between Doppler velocities and intensities in dark grains could be attributed to the fact that both intensities and Doppler velocities are the sum of several contributions along the LOS.

\subsection{Oscillations of individual structures}

Oscillations of individual dark grains. As we have already discussed in detail in Paper I, the Doppler velocity in dark grains exhibits a similar quasi-periodic behaviour as in mottles. In Fig. 5 we show the results of the wavelet analysis for the calculated Doppler velocity and line-center intensity variations (top panels of the figures) observed in the two dark grains "c" and "d" of Fig. 1. The middle left panels of the figures show the calculated power spectra as a function of time and period for the variations shown in the top panels (black in the gray scale represents high values of power). The contours represent the significance level of $95 \%$ which is obtained assuming a Poisson noise spectrum, while the cross-hatched 

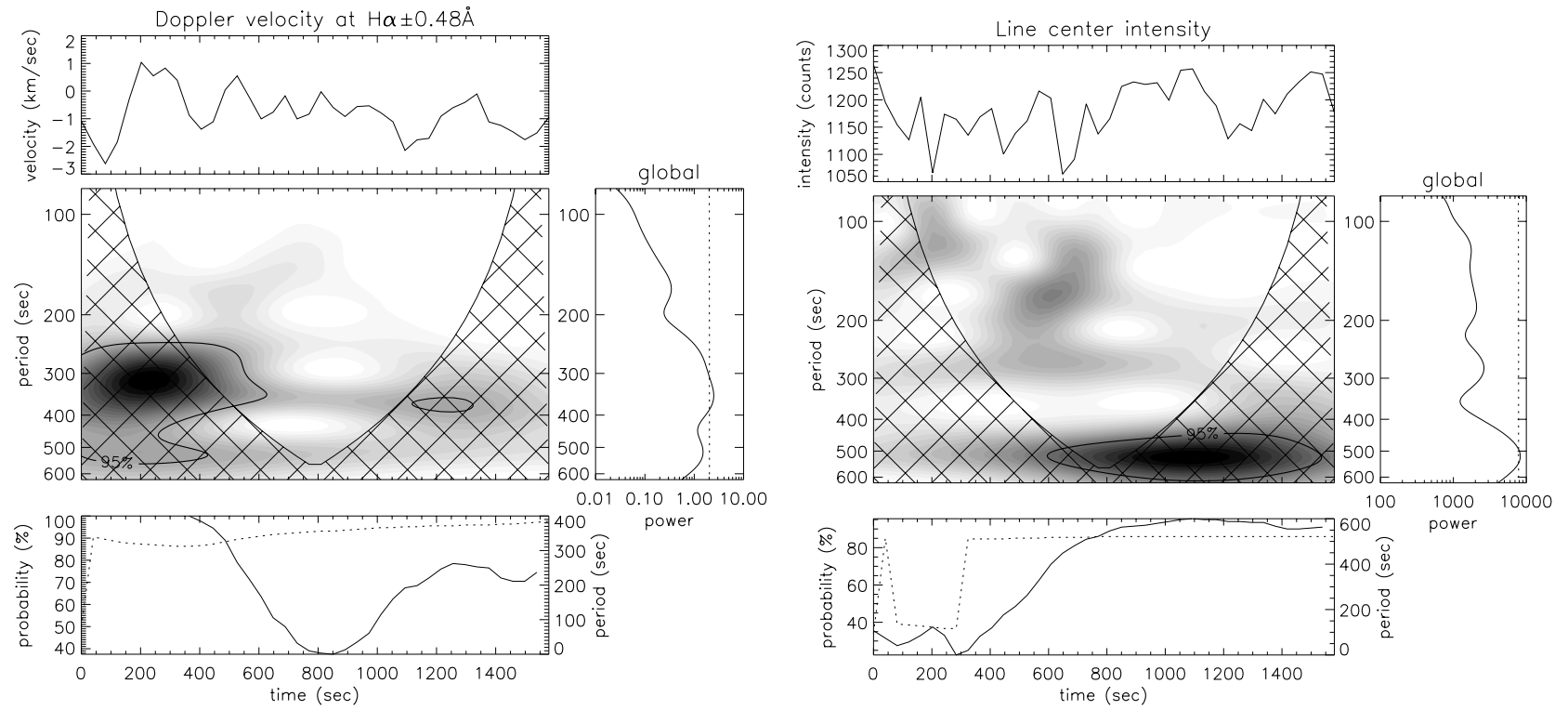

Fig. 6. Wavelet analysis results for the Doppler velocity (left column) and the $\mathrm{H} \alpha$ line-center intensity variations (right column) for the dark grain "d" of Fig. 1. For a description of the presented panels see caption of Fig. 5. The cut-off period is $562 \mathrm{~s}$ and the velocity global power spectrum has a peak period of $349 \mathrm{~s}$ with a global probability of $97.5 \%$.

regions indicate the $\mathrm{COI}$ regions which are subject to edge effects due to zero padding. The middle right panels show the global power spectrum, that is the average of the wavelet power spectrum over time. The dotted line - when present on the global power spectrum - indicates the equivalent global significance level of $95 \%$. Finally the lowest panels show the variations of the probability (solid line) obtained with the randomization method and the equivalent period of the maximum power peak (dotted line) for which this probability was calculated. The calculated probability using the randomization method is a better indication for the reliability of the obtained periods.

A wavelet analysis has been performed for the Doppler velocity at $\mathrm{H} \alpha \pm 0.48 \AA$ and $\mathrm{H} \alpha$ line-center intensity variations (Figs. 5 and 6) for the two dark grains marked as "c" and "d" in Fig. 1. It shows differences between the intensity and velocity power spectra - although the global power spectra for grain "c" are quite similar - and also a different behaviour between the two grains. However, both grains, show mostly periods around or in excess of 5 min with variable probabilities and considerable power close to $500 \mathrm{~s}$ which is, however, very close to the cut-off period of $562 \mathrm{~s}$ and therefore physically conspicuous.

Oscillations of individual dark mottles. For the study of the temporal behaviour of the velocity, derived with the cloud model, and the $\mathrm{H} \alpha$ line-center intensity along the mottles, which are presented in Fig. 2, we will restrict our analysis within the following location ranges: a) positions $4.9^{\prime \prime}$ to $10.8^{\prime \prime}$ for the mottle of region $\mathrm{A}$ and $\mathrm{b}$ ) positions 5.55" to $12.15^{\prime \prime}$ for the mottle of region B. The reason is that in these positions cloud velocities are derived for the whole time series. All distances are with respect to the feet of the mottles (see captions of Figs. 1 and 2 for definition).
In Figs. 7 and 8 we present the results of the wavelet analysis for the cloud velocity (Figs. 7a, 8a) and $\mathrm{H} \alpha$ line-center intensity variations (Figs. 7b, 8b) in the whole range of considered positions along the central axes of the two considered mottles of region A and B respectively (see caption of Fig. 7 for a description of figure panels). Although there is no discrimination in time for the results presented in both figures, we did a wavelet analysis instead of a Fourier analysis only for reasons of consistency (same method, same randomization process as in all previous and forthcoming results).

Again, the power analysis shows differences between the two mottles and considerable differences between velocity and intensity power spectra. However, the two considered individual mottles show quite clearly intensity and velocity oscillation periods close to $5 \mathrm{~min}$, but also a much fainter component of $\sim 3 \mathrm{~min}$. The periods obtained for the mottle in region $\mathrm{A}$ are in excess of $5 \mathrm{~min}$, while the periods obtained for mottle of region $\mathrm{B}$ are lower than $5 \mathrm{~min}$. The intensity power spectra suggest also a period close to $10 \mathrm{~min}$, like in the individual dark grains analysis, which is however very close to the cutoff period (thereby mostly in the COI) and hence its physical importance is doubtful.

\subsection{Oscillations of sets of dark mottles}

In Figs. 9 and 10 we present the wavelet analysis of the mean line-center intensity and cloud velocity variations of a large part of region A and B respectively, that contain a substantial number of dark mottles selected according to their intensity relative to the background (see Sect. 3). The considered mottles are those found at the top right corner of region A (around the selected cut) and all mottles of region B. The cut-off period is taken in both cases equal to $500 \mathrm{~s}$. In both images the cloud velocity variations are much smaller than the calculated cloud 

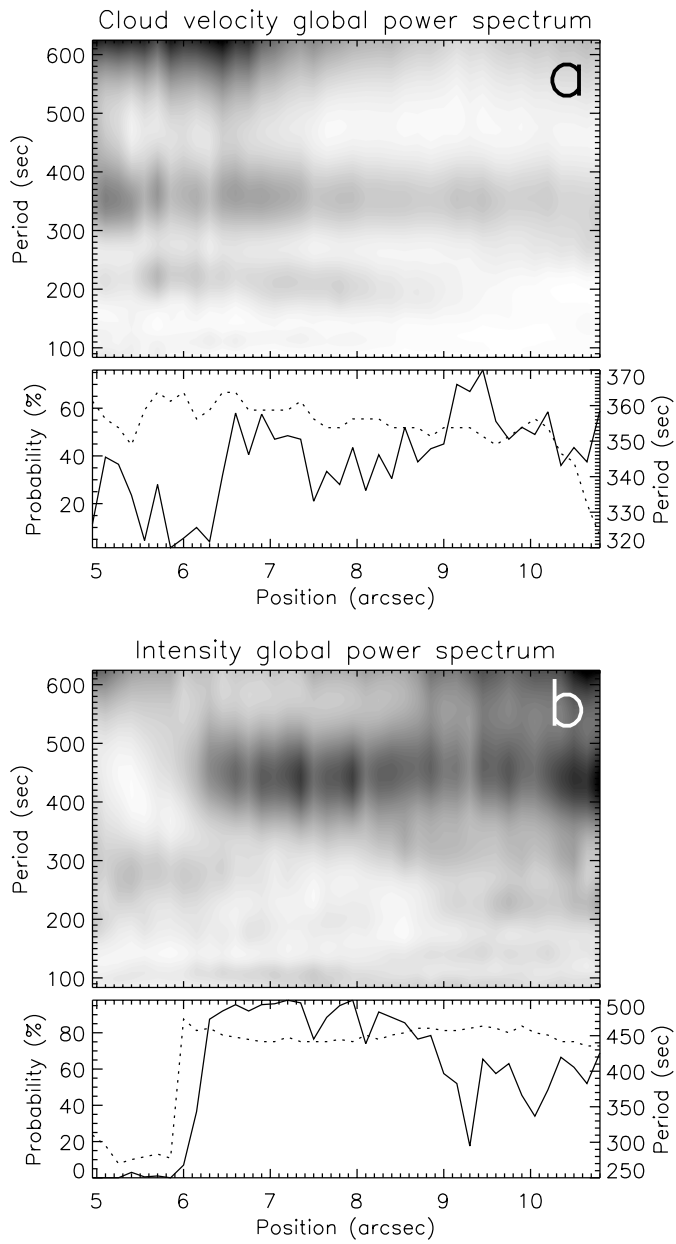

Fig. 7. Wavelet analysis results for a) the cloud velocity and b) the $\mathrm{H} \alpha$ line-center intensity variations at positions $4.9^{\prime \prime}$ to $10.8^{\prime \prime}$ along the mottle of region A of Fig. 2. The top panels show the global power spectrum as a function of period and position along the length of the mottle. The bottom panels show the variations of the global probability (solid line) obtained with the randomization method and the period (dotted line) of the maximum global power peak below the cut-off period (503 s) corresponding to this probability.

velocities of Fig. 2, since they represent an average of both positive and negative velocities. This is also clear from the velocity histogram of Fig. 5 of Paper I, which shows an almost Gaussian velocity distribution.

Like in oscillations of individual structures, presented in 5.2, there is a difference in behaviour between the two considered mottle regions. The velocity and intensity spectra of region A look quite similar (global power spectra are almost the same) but this is not the case for the respective power of region $\mathrm{B}$. There is a good match of the obtained spectra with the corresponding power spectra obtained for individual mottles and described in 5.2. We should note that the set of mottles in region $\mathrm{B}$ have periods close but lower than 5 min like the individual mottle of this region and the set of mottles of region A have periods larger than 5 min like the individual mottle of this region. There are also higher periods present, but these are extremely close to the cut-off value and hence physically doubtful, as well as a weaker signature close to the 3 min oscillation.
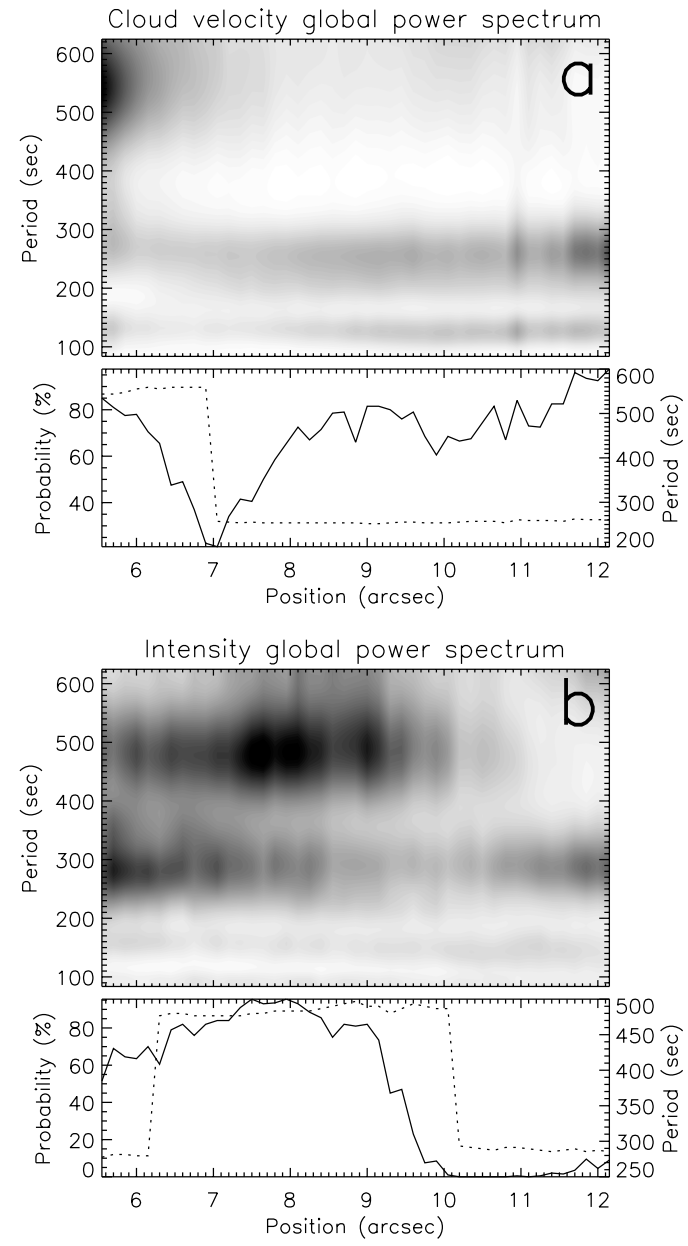

Fig. 8. Wavelet analysis results for a) the cloud velocity and b) the $\mathrm{H} \alpha$ line-center intensity variations at positions $5.55^{\prime \prime}$ to $12.15^{\prime \prime}$ along the mottle of region B of Fig. 2. For a description of the presented panels see caption of Fig. 7. The cut-off period is $562 \mathrm{~s}$.

\subsection{Oscillations of different areas of region A}

Mottles region oscillations. In Fig. 11 we present the results of the wavelet analysis for the calculated variations of the spatial averages of the line-center intensity and Doppler velocity at $\mathrm{H} \alpha \pm 0.48 \AA$ for the mottles area of region A shown in Fig. 3 (dotted gray contours). The Doppler velocity power spectrum shows two broad bands of oscillation periods around $170 \mathrm{~s}$ and $420 \mathrm{~s}$ with a width of $100 \mathrm{~s}$, while the line-center intensity spectrum shows a dominant period of $350 \mathrm{~s}$ (with a global probability of $42 \%$ ) and a much weaker oscillation around $150 \mathrm{~s}$. The cut-off period is $500 \mathrm{~s}$. Although not shown in this paper the mottles of region B, defined by applying a similar selection process, exhibits a similar behaviour, that means both a 3 min and a 5 min component.

Where do these two period components come from? The mottles region consists of a substantial number of mottles that oscillate (see Sects. 5.2 and 5.3) with a period larger than $5 \mathrm{~min}$ and are responsible for this period component seen in the spectra. However, there is also a substantial chromospheric background not eliminated by the averaging process which must be 

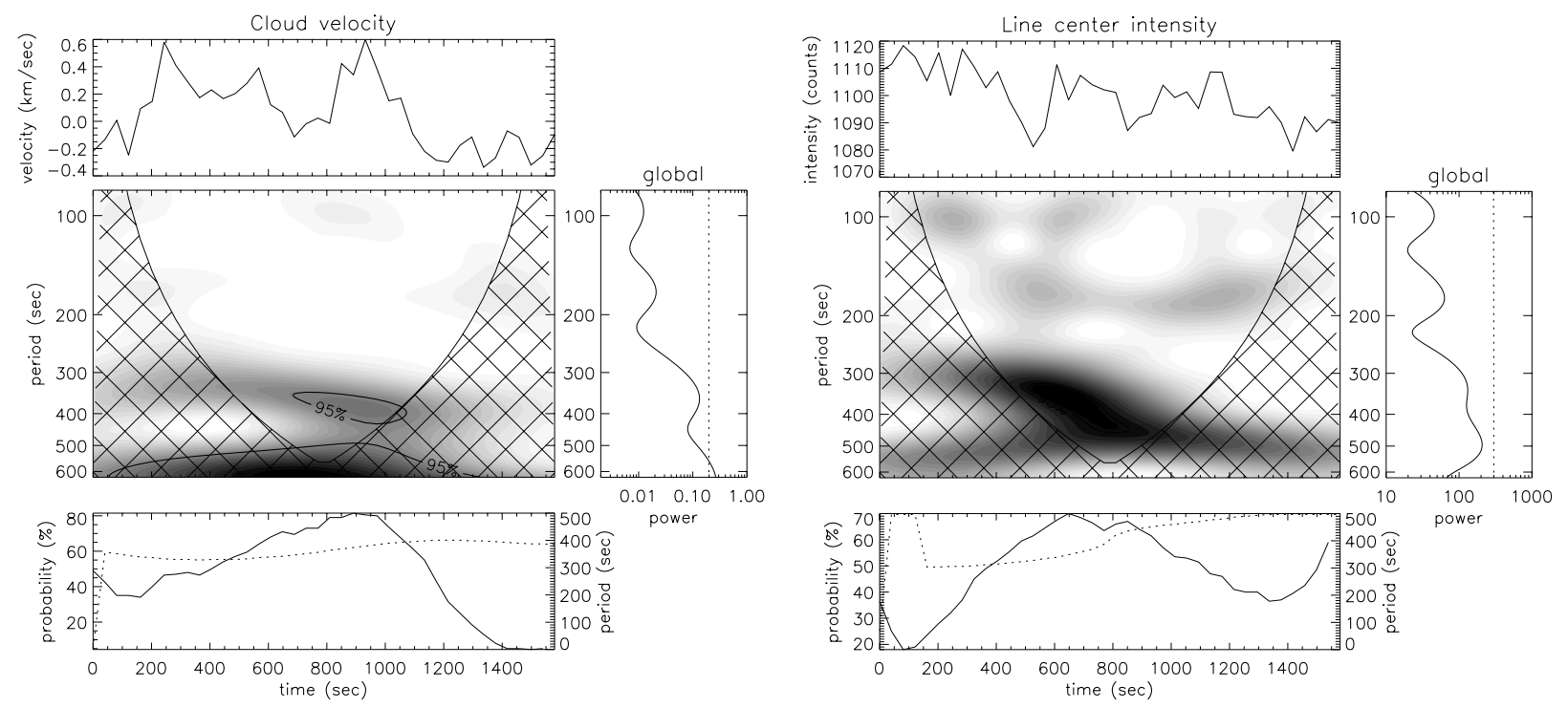

Fig. 9. Wavelet analysis results for the average cloud velocity (left column) and the average $\mathrm{H} \alpha$ line-center intensity variations (right column) for a part of region A containing dark mottles. For a description of the presented panels see caption of Fig. 5. The cut-off period is $500 \mathrm{~s}$. The respective peaks, below the cut-off period, of the global power spectra are at $361 \mathrm{~s}$ and $496 \mathrm{~s}$ with a corresponding global probability of $70 \%$ and $72.5 \%$
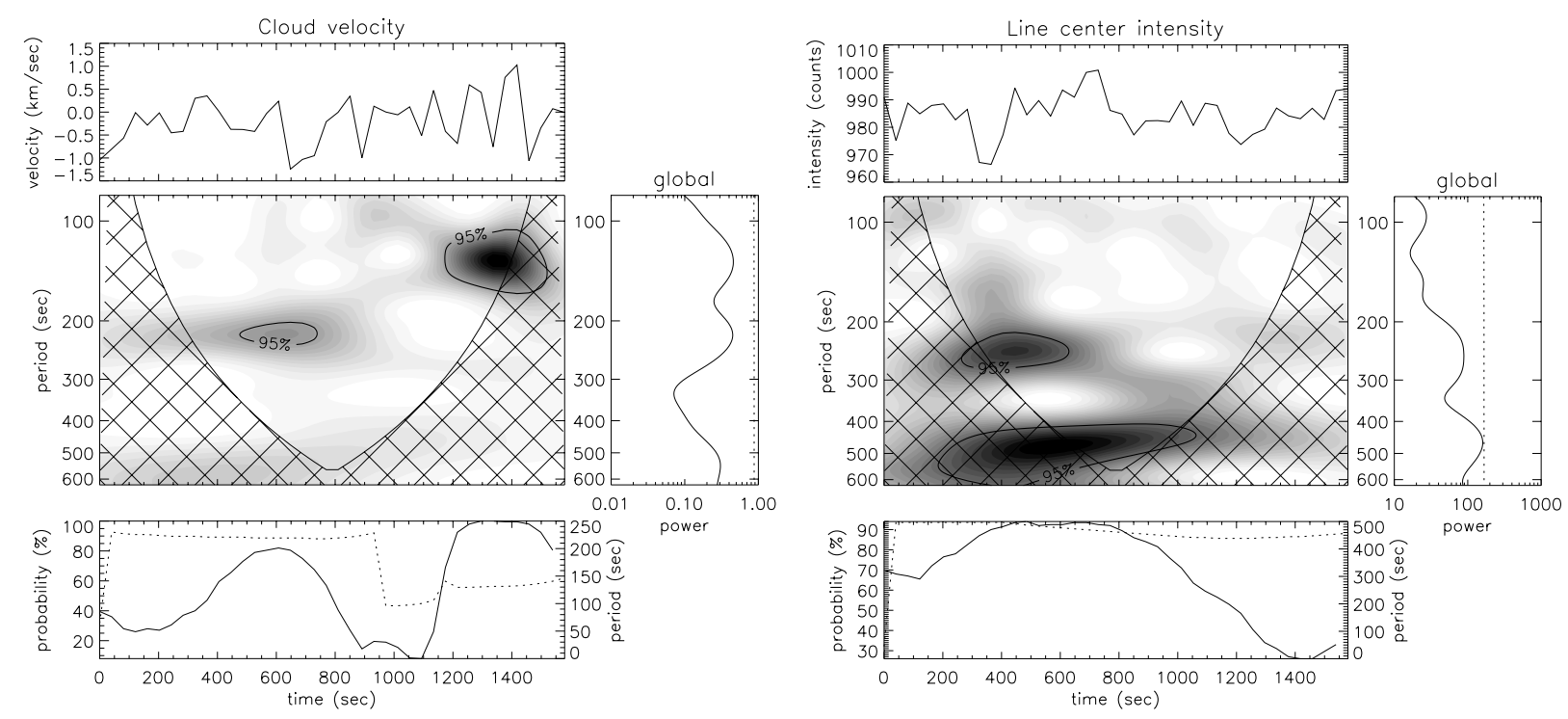

Fig. 10. Wavelet analysis results for the average cloud velocity (left column) and the average H $\alpha$ line-center intensity variations (right column) for a part of region B containing dark mottles. For a description of the presented panels see caption of Fig. 5. The cut-off period is $500 \mathrm{~s}$. The respective peaks, below the cut-off period, of the global power spectra are at $133 \mathrm{~s}$ and $463 \mathrm{~s}$ with a corresponding global probability of $35 \%$ and $98 \%$.

responsible for the low $\sim 3$ min period component present in the spectra. The selection process for this region results to an area which is structurally less coherent than the dark grains and grain-free areas, with several extended structures that morphologically evolve rapidly with time.

Oscillations of the grain-free region. We have calculated the spatial averages of line-center intensity and Doppler velocity at $\mathrm{H} \alpha \pm 0.48 \AA$ as a function of time for the grain-free area of region A shown in Fig. 3 (black contours). In Fig. 12 (left column) we present the power analysis of the Doppler velocity variations. The velocity power spectrum clearly shows a band of periods centered around $350 \mathrm{~s}$ with a very high probability close to $100 \%$. The global power spectrum has a maximum period of $347 \mathrm{~s}$ (below the cut-off period of $500 \mathrm{~s}$ ) with an estimated global probability of $\sim 100 \%$. The $\mathrm{H} \alpha$ line-center intensity power spectrum however shows several periods, mostly lower than $230 \mathrm{~s}$ with probabilities ranging from 5 to $70 \%$. There is also a fainter $350 \mathrm{~s}$ signature that persists for the whole duration of the time series. The maximum period of the global line-center intensity power spectrum is $228 \mathrm{~s}$ with an estimated global probability of $56 \%$. We comment on the difference between velocity and intensity power spectra in the discussion of this paper. 

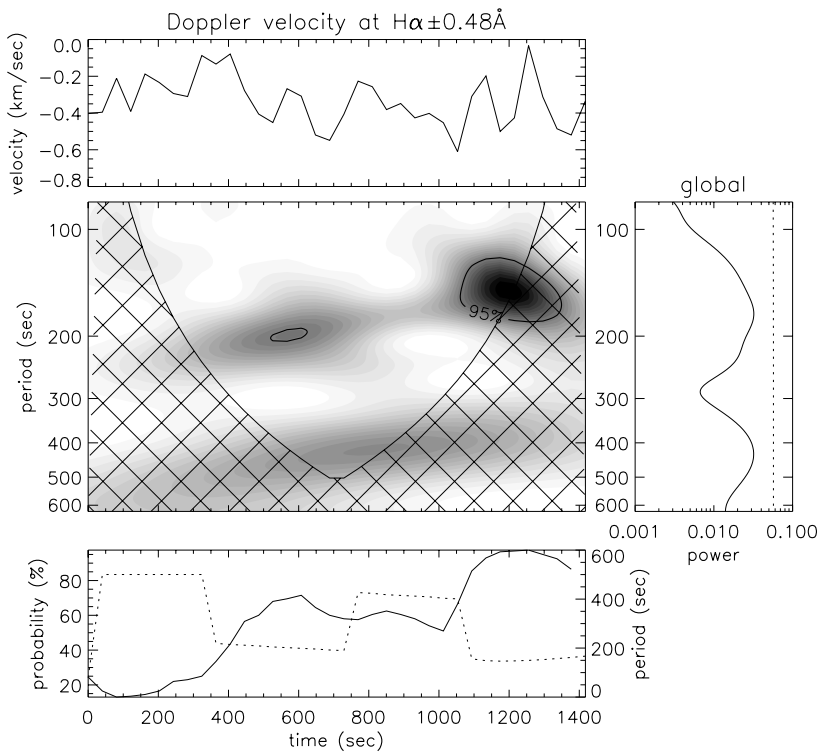
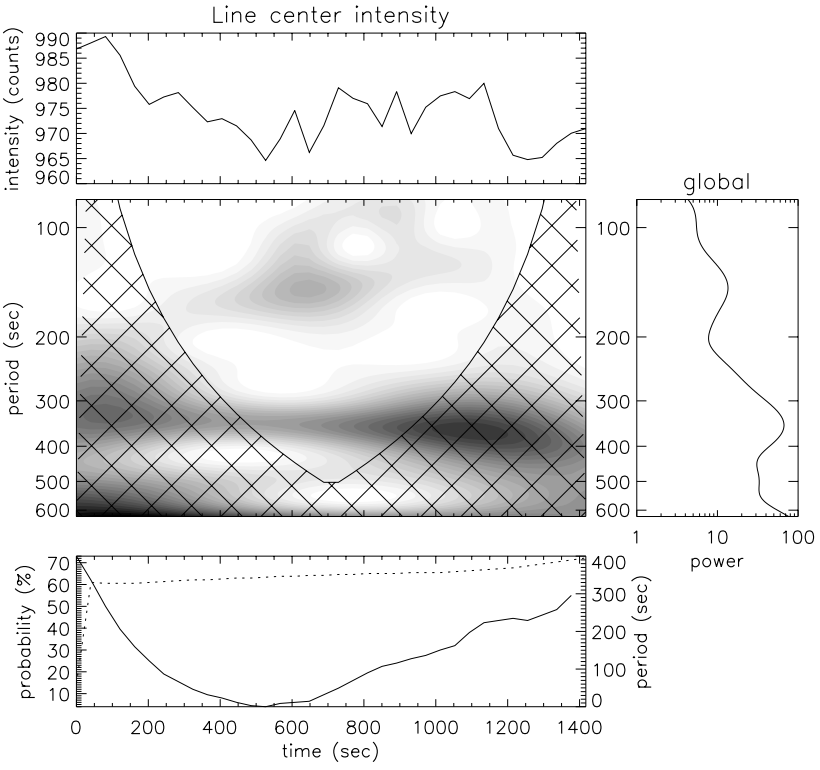

Fig. 11. Wavelet analysis results for the Doppler velocity (left column) and line-center intensity (right column) spatial averages of the mottles area shown in Fig. 3 (gray contours). For a description of the presented panels see caption of Fig. 5. The cut-off period is $500 \mathrm{~s}$.
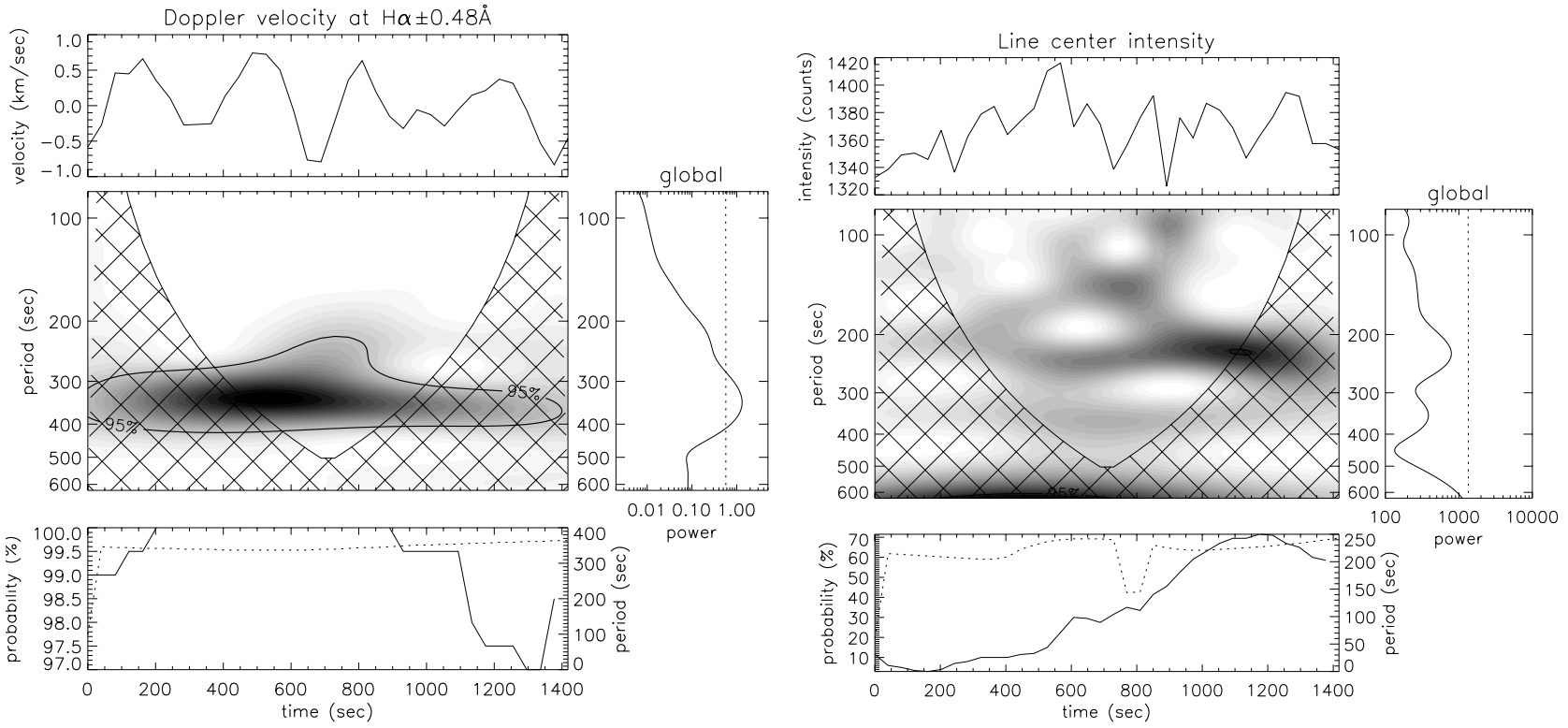

Fig. 12. Wavelet analysis results for the Doppler velocity (left column) and line-center intensity (right column) spatial averages of the grain-free area shown in Fig. 3 (black contours). For a description of the presented panels see caption of Fig. 5. The cut-off period is $500 \mathrm{~s}$.

The line-center intensity variations power spectrum presented above shows a multitude of signatures including the 3 min and 5 min ones, similar to the CI behaviour (Cram 1978; Lites 1993; Cauzzi 2000). We can thus stipulate that the grain-free region has internetwork-like characteristics.

Oscillations of dark grains region. In Fig. 13 we present the results of the wavelet analysis for the calculated variations of the spatial averages of line-center intensity and Doppler velocity at $\mathrm{H} \alpha \pm 0.48 \AA$ for the dark grains area of region A shown in Fig. 3 (white contours). Both power spectra show a broad peak around $300 \mathrm{~s}$, persisting almost for the whole duration of the time series and associated with very high probabilities close to $100 \%$. No other shorter period signatures are present. The line-center intensity global power spectrum has a maximum period of $274 \mathrm{~s}$ (below the cut-off period of $500 \mathrm{~s}$ ) and the Doppler global power spectrum a period of $289 \mathrm{~s}$ with an estimated global probability for both of $\sim 100 \%$.

The similarity of both power spectra is striking. A wavelet power analysis for other intensity fluctuations at $\pm 0.24 \AA$ and $\pm 0.48 \AA$ from the line center, which is not presented here, gives almost identical power spectra, with a global power 

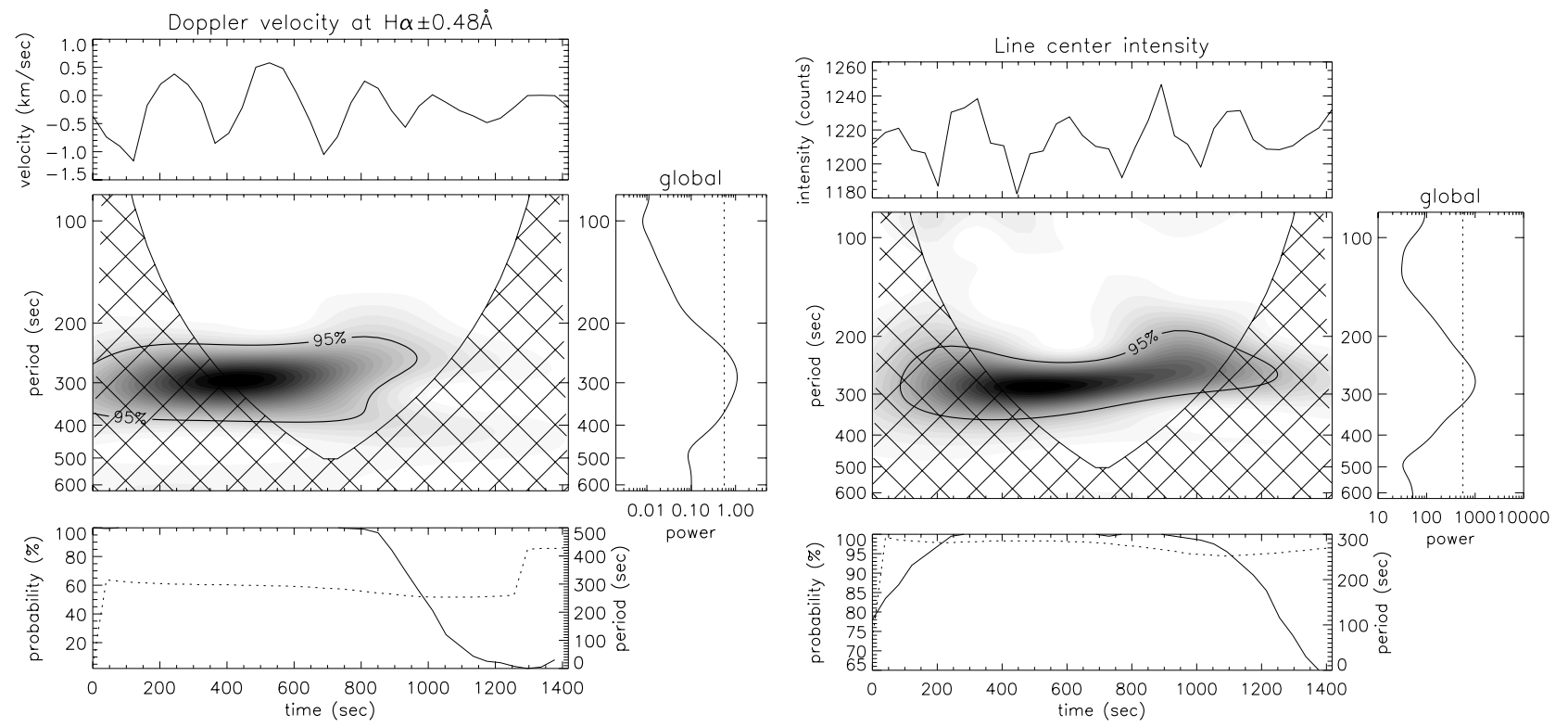

Fig. 13. Wavelet analysis results for the Doppler velocity (left column) and line-center intensity (right column) spatial averages of the dark grains area shown in Fig. 3 (white contours). For a description of the presented panels see caption of Fig. 5. The cut-off period is $500 \mathrm{~s}$.

period of $\sim 280 \mathrm{~s}$ and a global probability close to $100 \%$. Furthermore, it seems that there is a remarkable $\sim 90^{\circ}$ phase difference between Doppler velocity and line-center intensity variations. We should consider the following possibilities:

- dark grains are more likely vertical structures "anchored" in the photosphere and that we are probably observing the period signature of the flow along the structure. In Paper I we have suggested from the morphology and other physical characteristics of dark grains that they could be mottlelike structures vertical to the solar surface. Then, according to the proposed reconnection model for mottles, large bidirectional flows should often be present in dark grains like in dark mottles (see Paper I) resulting however to a small observed net component, since their main axis would be co-aligned with the LOS. If this is the case, the maximum downward (positive) velocity should correspond to the downflow phase along the whole structure, resulting to compression of the material at the feet of the structures and hence high intensities. Intensity becomes however highest later, during the reconnection phase, when opposite high bi-directional velocities occur, giving an almost zero net component along the structure, and resulting to higher compression at the feet of the structures (see also Fig. 2). Then as material cools down due to radiative looses at the feet and decompression at the top of the structures, indicated by increasing upward (negative) velocities, intensity drops and starts rising again as we gradually proceed to a new downflow phase;

- the dark grains region is just a region governed by $5 \mathrm{~min}$ p-modes. The $\mathrm{H} \alpha$ line-center intensity and Doppler velocity variations of the dark-grain region are the only ones showing a regular 5 min wavetrain which is known to be produced by the p-mode pattern but very intermittently in space and time. However, being both at the right moment and place for a maximum amplitude p-mode interference wavetrain for such a long time is very unlikely.

Dark grains and the grain-free area are found inside the low magnetic flux plage region. However, despite their spatial proximity the line-center intensity variations exhibit a totally different oscillatory behaviour. While the line-center intensity of the grain-free region oscillates with periods closer to $3 \mathrm{~min}$, dark grains have a period of $\sim 5 \mathrm{~min}$, which is similar to the period obtained for dark mottles.

\section{Validity of spectral analysis for spatial averages}

For the wavelet analysis of areas of region A (see Sect. 5.4), we have considered the temporal variations of the spatial averages of intensities or Doppler velocities of the corresponding areas of Fig. 3. It is well known that the $\mathrm{H} \alpha$ line, a chromospheric line, contains also a significant photospheric component (see Schoolman 1972). One may argue that spatial averaging of signals before the spectral analysis rather than spectral analysis at each pixel of the image followed by spatial averaging of the obtained power spectra will suppress spatially relatively incoherent and small scale signatures like the $3 \mathrm{~min}$ oscillations, leaving the large scale 5 min photospheric contributions intact. Our analysis however shows that this is not absolutely true: the line-center average intensity power spectrum of Fig. 12 for the grain-free region shows that, although there are faint 5 min photospheric signatures present, the dominant component of the oscillations is the chromospheric one around 3 min. In Fig. 14 we show how the global power spectrum of spatial averaged intensity fluctuations of the grain-free region changes when we consider the line-center intensity and intensities at $\pm 0.24 \AA$ and $\pm 0.48 \AA$ from the line center (wings of the profile). The line-center and $\pm 0.24 \AA$ global power spectra (solid and dotted curve) still show a more dominant chromospheric period component closer to 3 min which seems to shift 


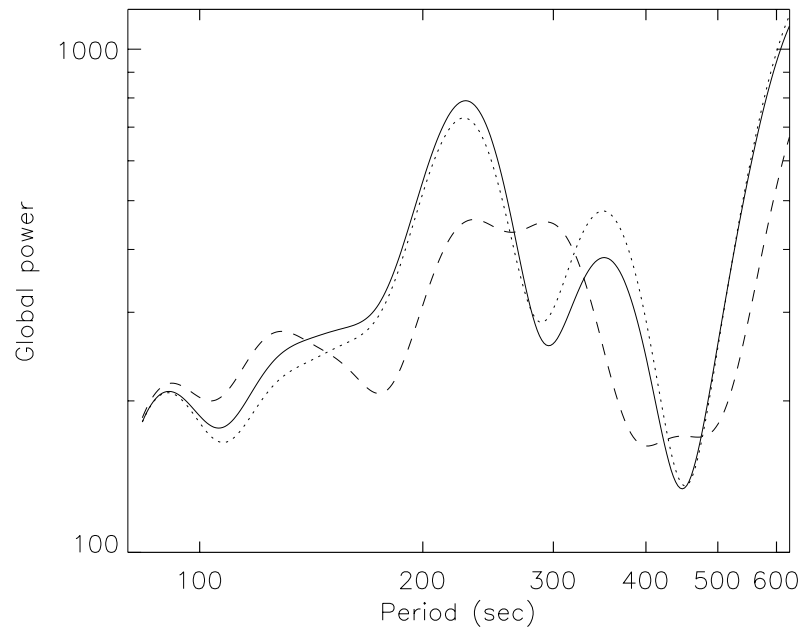

Fig. 14. The global power spectrum of the spatial averages over the grain-free area shown in Fig. 3 (black contours) of the linecenter intensity (solid line), intensity at $\mathrm{H} \alpha \pm 0.24 \AA$ (dotted line) and $\mathrm{H} \alpha \pm 0.48 \AA$ (dashed line). There is a clear shift from chromospheric ( $\sim 3 \mathrm{~min})$ to photospheric $(\sim 5 \mathrm{~min})$ signatures as we move to deeper layers (towards the wings of the profile).

- as expected - towards a 5 min photospheric component as we move towards the wings and hence to deeper layers of the atmosphere. This is yet another indication that the spatial averaging of line-center intensity signals does not destroy all information about the oscillations and preserves most of their basic physical characteristics.

On the other hand, doing a spectral analysis of temporal variations at each pixel of an area of interest before averaging would be risky, since pixels do not always correspond on the same part of a structure from one image to the other. As we have discussed in Paper I most of the observed structures and in particular mottles evolve spatially with time, changing often size and direction. Thus, a temporal analysis at each pixel of interest would be meaningless unless it is possible to be carefully traced in time. This has been done only for the oscillations of individual structures presented in Sect. 5.2, but obviously it is impossible for large regions.

\section{Discussion and conclusions}

A wavelet analysis method, permitting the investigation of any present periodicity localized in time, is used to investigate oscillations of individual dark mottles and grains, and areas containing or not such structures. In Paper I we have suggested that dark grains are essentially the same structures as dark mottles their only difference being their respective inclinations relative to the solar surface; according to the presented there morphological study, we suggested that dark grains are structures nearly vertical to the solar surface, while mottles are inclined relative to the LOS. If this is the case what are the new arguments that the present study provides? They are as follows:

1. Although differences do exist in the obtained periodicities of intensity and velocity variations, individual mottles, regions of mottles, individual grains and regions of grains show a most prominent period in the $5 \mathrm{~min}$ range $(3.3 \mathrm{mHz})$. This is not a conclusive argument, it provides, however, an indication for the similarity of dark mottles and grains.

2. Intensity and Doppler velocity power spectra of the grain region are strikingly similar. They all show a same unique period $\sim 5$ min with a high probability close to $100 \%$, which could be explained as the period signature of flows along a vertical structure "anchored" in the photosphere. However, the presence of a maximum amplitude p-mode interference wavetrain in the grain region, although highly unlikely, cannot be for the moment excluded.

3. Dark grains and the grain-free area both define the low magnetic flux plage region, with the latter exhibiting internetwork-like characteristics. However, despite their spatial proximity the line-center intensity variations of the two regions exhibit a totally different oscillatory behaviour, with the grain-free one oscillating with periods closer to $3 \mathrm{~min}$ and the dark grains one with a period of $\sim 5 \mathrm{~min}$, which is similar to the period obtained for dark mottles.

A comparison of the periods obtained for the two considered individual dark mottles (mottle A in region A and mottle B in region $\mathrm{B}$ ) shows that although both have their maximum power in the 5 min range there are some differences between them. This difference is even more apparent in the wavelet analysis of the average intensities and cloud velocities of large parts of regions $\mathrm{A}$ and $\mathrm{B}$ which, however, preserve the period signatures observed in the corresponding individual mottles. Individual dark grains "c" and "d" show also different periods. It seems that the explanation is differences in the magnetic field topology which can influence the oscillation pattern. While mottles in region B stand in a chain defining a network boundary where the magnetic field (although not resolved in the MDI magnetograms) has small inclination, mottles of region A lie in the periphery of a plage where the magnetic field is more inclined. Grain "c" which also lies close to the plage periphery, shows periods similar to the periods of mottle A, while grain "d", which lies further from the plage periphery, shows almost the same period for the intensity variations as the dark grain region average. Numerical calculations carried out by Rosenthal et al. (2002) although under very idealized physical conditions have shown that in regions where the field is close to the vertical the waves propagate upward largely unaffected by the field. On the other hand in regions where the field is inclined to the vertical, waves could be reflected at a surface whose altitude is highly variable having consequences on the oscillatory processes. It is not only the geometry but also the magnitude of the local magnetic field that affects the observed periods. Periods found in region A seem to be in general higher than periods found in region $\mathrm{B}$ in accordance with previous findings that oscillation periods are larger in higher magnetic regions due to a rise of the acoustic cut-off period because of gravity (Bel \& Mein 1971; Blondel 1971).

We found a clear correlation between redshifts and high intensities for mottles. As reported by Gallagher et al. (1999) and Hansteen et al. (2000) this correlation exists also for the whole $\mathrm{CB}$ observed in transition region lines. On the other 
hand, no correlation is found between intensities and velocities of the dark grains. If these structures are indeed mottles standing vertically on the solar surface then this lack of correlation can be attributed to the fact that both quantities are the sum of several contributions along the LOS.

Furthermore, an inspection of all obtained wavelet spectra shows the following general characteristics:

1. Velocity (cloud and Doppler) and intensity variations are very often bursty and intermittent, occurring only for parts of the full time series. However, there are periods that persist for the whole duration of the analyzed time series, with often varying significance and probability.

2. Velocity variations, as noticed before (Cram 1978; Hansteen et al. 2000; Banerjee et al. 2001), seem to be dominant over intensity variations, showing much clearer and better confined period signatures. They show a large range of periods from 100 to $500 \mathrm{~s}$ with the dominant one centered around $\sim 300 \mathrm{~s}$. Intensity oscillations are also centered around $\sim 300 \mathrm{~s}$, but are less prominent. This is probably also due to the fact that the visibility of mottles does not change significantly with time. Tsiropoula et al. (1994) investigating cross-correlation functions of dark mottles found that the velocity shows much more important changes with time than the intensity.

3. A 3 min oscillations signature is present in almost all linecenter intensity spectra, but never is the dominant one, expect for the grain-free region; this is also the case for some of the velocity spectra.

4. An intermittent signature of $100 \mathrm{~s}(10 \mathrm{mHz})$, reported before in literature (Hansteen et al. 2000), is present in almost all intensity spectra and some of the velocity ones. It lies close to the Nyquist frequency of $12.3 \mathrm{mHz}$ (81 s) that we derive from the observational cadence of our data.

5. Intensity and velocity power spectra show very often remarkable differences.

Detected oscillation periods above the $95 \%$ confidence level are quite reliable. We have, however, also reported periodicities with lower probabilities, which are consistent with the findings of other authors. Some of these low probabilities are probably due to the observation time interval, which is rather short (27 min). As the wavelet analysis suffers from edge effects at both ends of the time series, a longer observing time would have increased the number of cycles outside the COI, as well as the maximum detectable period, while a higher cadence would have been useful in making definitive statements about the shorter periods.

Let us comment on the difference between several $\mathrm{H} \alpha$ linecenter intensity and Doppler velocity power spectra. Intensity is a complicated result of both dynamics and radiative transfer effects (Cram 1978). Intensity fluctuations of $\mathrm{H} \alpha$ wings, usually formed in depths with small velocity gradients and LTE conditions, reflect fluctuations of the source function and hence temperature. For the line-center intensity, non-LTE effects are important and fluctuations respond to temperature, density and even velocity changes. The Doppler velocity, however, which contains information concerning the last scattering, is more sensitive to local conditions than intensity and is a fairly good diagnostic for velocity. A detailed radiative transfer analysis is needed to resolve the velocity stratification within the structure and even then this analysis is extremely dependent on the adopted model of the structure or atmosphere. Furthermore, the existence of some low periods only in intensity and not velocity power spectra of individual structures could be attributed to mistakes in the spatial co-alignment with time of structures with strong spatial intensity variations. On the other hand, velocities usually have similar phases in neighboring pixels and hence are less affected by co-alignment mistakes.

The present analysis unfortunately suffers from a) lack of phase information $b$ ) reliable statistics for individual structures and c) low duration of the observations. Phase information is necessary for a detailed study of the propagation characteristics especially in the dark grain region where an ambiguity exists on whether the observed oscillations are flow signatures along vertical structures or p-modes. The observed V-I phase difference of $90^{\circ}$ is not enough by itself to give conclusive arguments. In this context observations in more lines, formed at different depths, are needed for a correct interpretation of the results. As far as statistics is concerned, although region averages provide some indications and general trends, measuring a lot of individual structures or taking averages from a longer duration data set, obtained with less variable seeing conditions, is necessary to definitely confirm the presented oscillatory behaviour.

Mottles and dark grains, which constitute part of the chromospheric fine structure, could be related to the heating of the chromosphere and lower corona. Thus a better understanding of their properties will undoubtedly shed much light on the questions associated with the dynamics and heating of the solar atmosphere.

Acknowledgements. The THEMIS telescope is operated on the island of Tenerife by CNRS-CNR in the Spanish Observatorio del Teide of the Instituto de Astrofísica de Canarias. We would like to thank S. Regnier (PI of the project) and C. Briand (observer) for providing us the observations. We would also like to thank R. J. Rutten for many useful discussions and comments. Wavelet software was provided by $\mathrm{C}$. Torrence and G. Compo and is available at URL: http://paos.colorado.edu/research/wavelets/. This research is part of "Programme d'Actions Intégrées Francohelléniques PLATON". KT and PM are members of the European Solar Magnetism Network (ESMN) supported by the EC through the RTN programme.

\section{References}

Banerjee, D., O'Shea, E., Doyle, J. G., \& Goossens, M. 2001, A\&A, 371,1137

Bel, N., \& Mein, P. 1971, A\&A, 11, 234

Bhatnagar, A., \& Tanaka, K. 1972, Sol. Phys., 24, 87

Blondel, M. 1971, A\&A, 10, 342

Bradley, J. V. 1968, Distribution-Free Statistical Tests (New York: Prentice-Hall), 68

Cauzzi, G., Falchi, A., \& Falciani, R. 2000, A\&A, 357, 1093

Cram, L. E. 1978, A\&A, 70, 345

Damé, L., Gouttebroze, P., \& Malherbe, J.-M. 1984, A\&A, 130, 31 
Deubner, F.-L., \& Fleck, B. 1990, A\&A, 228, 506

Fludra, A. 2001, A\&A, 368, 639

Foukal, P. 1971, Sol. Phys., 19, 59

Gallagher, P. T., Phillips, K. J. H., Harra-Murnion, L. K., Baudin, F., \& Keenan, F. P. 1999, A\&A, 348, 251

Hansteen, V. H., Betta, R., \& Carlsson, M. 2000, A\&A, 360, 742

Krijger, J. M., Rutten, R. J., Lites, B. W., et al. 2001, A\&A, 379, 1052

Lites, B. W., Rutten, R. J., \& Kalkofen, W. 1993, ApJ, 414, 345

Muglach, K. 2003, A\&A, 401, 685

Nemec, A. F., \& Nemec, J. M. 1985, AJ, 90, 2317

Rosenthal, C. S., Bogdan, T. J., Carlsson, M., et al. 2002, ApJ, 564, 508
Rutten, R. J. 1999, in 3rd Advances in Magnetic Fields and Oscillations, Sol. Phys. Euroconf., ed. B. Schmieder, A. Hofmann, \& J. Staude, ASP Conf. Ser., 184, 181

Schoolman, S. A. 1972, Sol. Phys., 22, 344

Schrijver, K. 1989, Sol. Phys., 122, 193

Torrence, C., \& Compo, G. P. 1998, Bull. Amer. Meteor. Soc., 79, 61

Tsiropoula, G. 2000, New Astron., 5, 1

Tsiropoula, G., Alissandrakis, C. E., \& Schmieder, B. 1994, A\&A, 290, 285

Tziotziou, K., Tsiropoula, G., \& Mein, P. 2003, A\&A, 402, 361

von Uexküll, M., \& Kneer, F. 1995, A\&A, 294, 252 